\title{
Neural mechanisms of interleaved practice that support long-term retention of motor skill in stroke: translational research perspective
}

\author{
Taewon Kim (iD https://orcid.org/0000-0001-6870-4379
}

\begin{abstract}
A fifteen million people suffer stroke worldwide each year according to the World Health Organization. Stroke is the leading cause of long-term disability with motor deficit being the most common deficit after stroke (World Health Organization, 2002). Because neural plasticity is believed to be central to the acquisition of motor skill and learning in the intact brain as well as relearning in the damaged brain, recent advances in understanding, experience-dependent neural plasticity can inform ongoing development and adaptation of contemporary therapeutic interventions to improve recovery and rehabilitation (Dimyan and Cohen, 2011; Winstein and Kay, 2015). Unfortunately, identification of theory-driven best-practice has been hampered because many clinical trials are implemented during the chronic rather than acute stage after stroke, a period during which plastic changes supporting recovery are less likely to emerge. These trials are further hindered by despite the use of novel technologies, such as robotic-guided training, being used either in isolation or as an adjunct to contemporary behavioral intervention, to induce greater dosing (Krakauer, 2006).
\end{abstract}

A fundamental principle to facilitate motor recovery in rehabilitation is extensive a practice that patients need for everyday functions. For the neurorehabilitation setting, a promising avenue to boost neuronal excitability and strengthen functional connectivity in neurologically impaired patients is practicing motor skills for learning (Maier et al., 2019). Indeed, motor learning mechanisms are interacted with motor recovery process and these interactions can enhance greater neural plasticity reflected in superior neurore- habilitation outcomes including long-term retention of motor skills and motor recovery (Krakauer, 2006; Maier et al., 2019).

Here, the focus is on fresh evidence illustrating the efficacy of learning multiple skill memories in an interleaved practice (IP) format that is in keeping with the recent mantra that much of skill learning occurs offline (Wright et al., 2016). In this regard, few questions the importance of extensive practice for learning, how practice is organized has been reported to be critical for optimizing skill acquisition. The impact of inducing greater contextual interference $(\mathrm{CI})$ during training is a practice scheduling protocol that has garnered greater $\mathrm{CI}$ is often engineered during motor skill acquisition through practice of multiple motor tasks in an IP format. Conversely, repetitive practice (RP) creates significantly less interference because it involves the repeated execution of the same motor task before facing subsequent practice with other motor tasks. What is most striking during the test trials is that prior exposure to RP usually leads to significant forgetting. In contrast, individuals trained via IP exhibit stable or even enhanced performance, often referred to as offline gain, suggesting that IP is important for facilitating postpractice consolidation, a process reported to be central to the establishment of a robust motor memory (Kim et al., 2018; Kim et al., 2021). In this letter, I highlight critical research insights in motor learning in stroke based on the neural mechanism of IP format.

The study by Schweighofer et al. (2011) entitled "Mechanism of contextual interference effect in individuals poststroke" published in Journal of Neurophysiology is indeed an enlightening study 
that examined the impact of IP on motor learning in poststroke. Schweighofer et al. (2011) found that stroke patients trained visuomotor learning task via IP exhibited stable, but significant forgetting occurred in stroke patients trained via RP in retention test that was administrated $24 \mathrm{hr}$ after the completion of practice. This finding was initial work to investigate theocratical and conceptual idea of IP benefits on long-term retention of motor learning applied to stroke patients.

Neural imaging evidence during IP and RP supported that IP instigates the recruitment of a broader network of neural regions (sensorimotor and frontoparietal networks). Specifically, differential cost of movement preparation reflected in the increase in attention demand is accompanied by distinct neural recruitment strategies during RP and IP formats. Therefore, increased CI such as IP during training was associated with greater recruitment of neural regions, in particular, the relative contribution of the primary motor cortex (M1) as well as both lateral premotor and medial premotor regions increased dramatically across IP but not RP (Lin et al., 2018). Moreover, M1 excitability at the time of retention is greater following IP (Lin et al., 2011) and stronger task-related functional connectivity later in training between the right dorsal premotor area and M1 during IP is associated with the subsequent retention advantage exhibited by IP participants (Lin et al., 2013).

Despite the robust behavioral enhancements associated with strong neural imaging evidence for motor learning in IP structure (Lin et al., 2011; Lin et al., 2013; Lin et al., 2018; Wright et al., 2016), there has not been examined with respect to neural mechanism underlying advantage of IP on motor learning and recovery in neurorehabilitation. Surprisingly, there is no study examined impact of IP on motor learning in stroke combined with brain imaging techniques. This knowledge gap is surprising given that impact of IP benefits has been hailed as a potential intervention for motor deficits particularly neurological-related impairments. Going forward, future studies need to be done to examine how IP aid changes in stroke brain states impacting on motor learning, and how this learning outcomes correlate to the success of motor recovery using brain imaging techniques.

\section{CONFLICT OF INTEREST}

No potential conflict of interest relevant to this article was reported.

\section{REFERENCES}

Dimyan MA, Cohen LG. Neuroplasticity in the context of motor rehabilitation after stroke. Nat Rev Neurol 2011;7:76-85.

Kim T, Chen J, Verwey WB, Wright DL. Improving novel motor learning through prior high contextual interference training. Acta Psychol 2018; 182:55-64.

Kim T, Kim H, Wright DL. Improving consolidation by applying anodal transcranial direct current stimulation at primary motor cortex during repetitive practice. Neurobiol Learn Mem 2021;178:107365.

Krakauer JW. Motor learning: its relevance to stroke recovery and neurorehabilitation. Curr Opin Neurol 2006;19:84-90.

Lin $\mathrm{CH}$, Chiang MC, Knowlton BJ, Iacoboni M, Udompholkul P, Wu AD. Interleaved practice enhances skill learning and the functional connectivity of fronto-parietal networks. Hum Brain Mapp 2013;34:1542-1558.

Lin $\mathrm{CH}$, Knowlton BJ, Chiang MC, Iacoboni M, Udompholkul P, Wu AD. Brain-behavior correlates of optimizing learning through interleaved practice. Neuroimage 2011;56:1758-1772.

Lin CH, Yang HC, Knowlton BJ, Wu AD, Iacoboni M, Ye YL, Huang SL, Chiang MC. Contextual interference enhances motor learning through increased resting brain connectivity during memory consolidation. NeuroImage 2018;181:1-15.

Maier M, Ballester BR, Verschure PF. Principles of neurorehabilitation after stroke based on motor learning and brain plasticity mechanisms. Front Syst Neurosci 2019;13:74.

Schweighofer N, Lee JY, Goh HT, Choi Y, Kim SS, Stewart JC, Lewthwaite $\mathrm{R}$, Winstein CJ. Mechanisms of the contextual interference effect in individuals poststroke. J Neurophysiol 2011;106:2632-2641.

Winstein CJ, Kay DB. Translating the science into practice: shaping rehabilitation practice to enhance recovery after brain damage. Prog Brain Res 2015;218:331-360.

World Health Organization. The world health report 2002: reducing risks, promoting healthy life. Geneva (Switzerland): World Health Organization; 2002.

Wright DL, Verwey WB, Buchanen JJ, Chen J, Rhee J, Immink M. Consolidating behavioral and neurophysiologic findings to explain the influence of contextual interference during motor sequence learning. Psychon Bull Rev 2016;23:1-21.

Division of Stroke \& Vascular Neurology, Department of Neurology, Duke University School of Medicine, Durham, NC 27710, USA Email: taewon.kim@duke.edu 